\title{
Pain management in a low income setting
}

\author{
V. Camarda 1 , M. Gallieni 2 , S. K. Brahmochary 3 , A. Doneda 4 , G. Landoni 1 , L. \\ Beretta 1
}

1 Vita-Salute San Raffaele University - Milan (Italy), 2 Department of Clinical and Biomedical Sciences

"Luigi Sacco", University of Milan, Italy. - Milan (Italy), 3 Institute for Indian Mother \& Child - Kolkata (India),

4 Project for People - Milan (Italy)

\begin{abstract}
Background:
Pain relief is a human right, as stated by the International Association for the Study of Pain (IASP) in 2004 (1). Nevertheless, in a low income setting, the majority of people does not receive appropriate treatment for pain and the problem is not strictly related to costs. Our project "Pain management in low income setting", has been carried out in a rural area of south Kolkata, West Bengal, in April and May 2017, in collaboration with Project for People and Institute for Indian Mother and Child (IIMC) with the aim of investigating chronic pain and introducing a chronic pain management system.
\end{abstract}

\section{Methods:}

The project was: educational (through lessons to health workers and the development of algorithms for clinical practice); on the field (aiding in daily practice with patients affected by chronic pain); scientific (gathering epidemiological patients' data).

\section{Results and Discussion:}

We report preliminary data from the first mission, aimed at establishing the project. We assessed a sample of 40 out of thousands patients consulting our outdoor clinic in Kolkata, the majority of which were female (75\%). The mean age was 50 (range 18-104); Osteoarthritis and nonspecific articular pain where the most represented causes of pain. Average Numeric Rating Scale for pain was 6,9 with moderate to severe functional reduction (mean quality of life scale was 5,4 ). Pain was usually endured for several months or years (up to ten years). The patients were treated according to the WHO model of cancer pain management, but with the limitation of the locally available drugs (NSAID, paracetamol, pregabalin and dexamethasone); opioid were not used in this setting. Intramuscular and oral were the preferred route of administration; we also recommended physical therapy and behavioral changes.

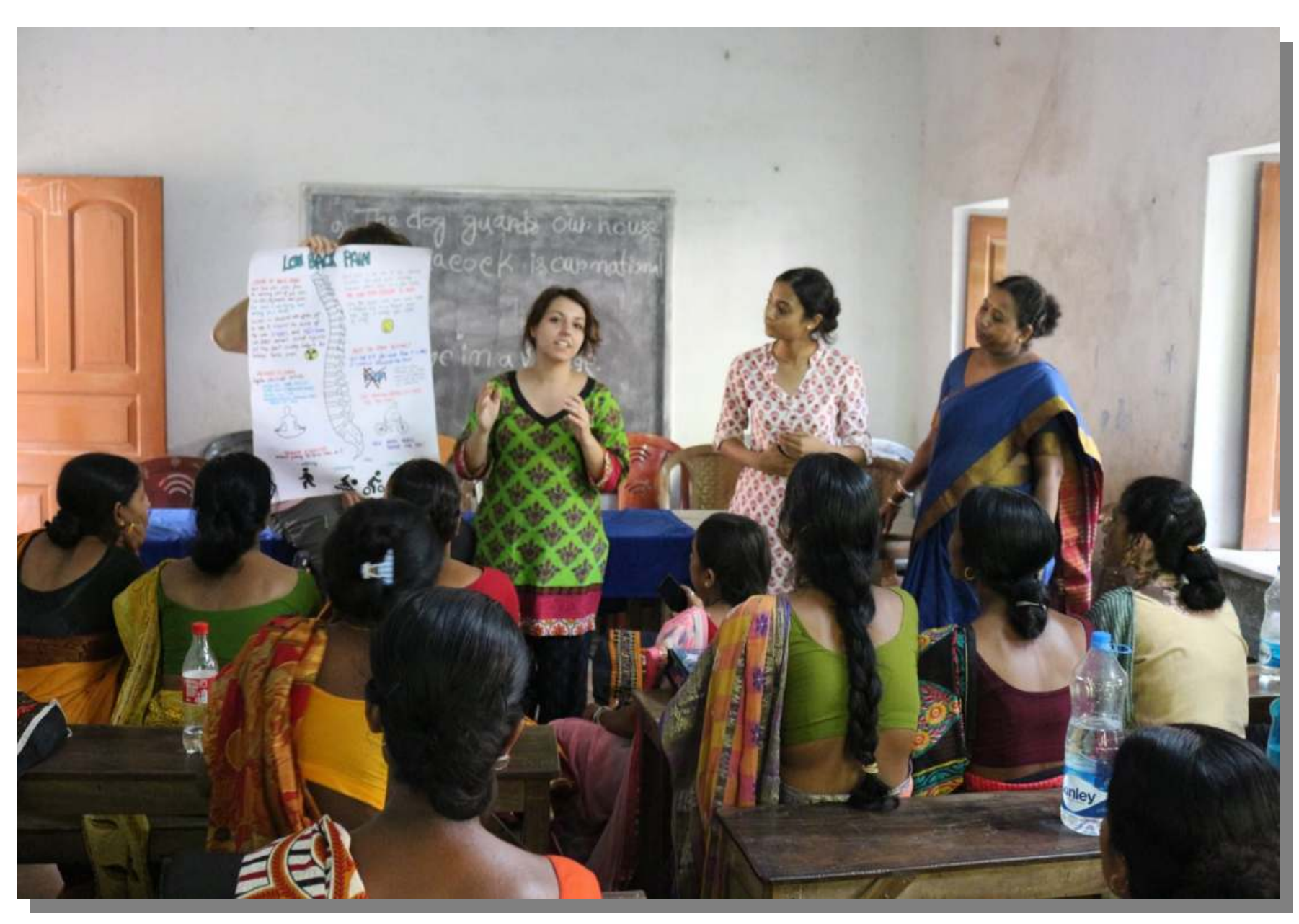

\section{Conclusions:}

Pain is an important problem in all countries, including areas with limited accessibility to health care, and can severely affect patient's quality of life. People in low income setting have little or no palliative care or pain relief. Conditions such as malnutrition, infections, and parasitic diseases are prevalent, and pain is often not considered a priority of health care policies. Ensuring access to medicines as well as adequate training of health personnel are among the main objectives for achieving adequate pain management also in low income settings.

"Poverty is not just a lack of money; it is not having the capability to realize one's full potential as a human being" Amartya Sen

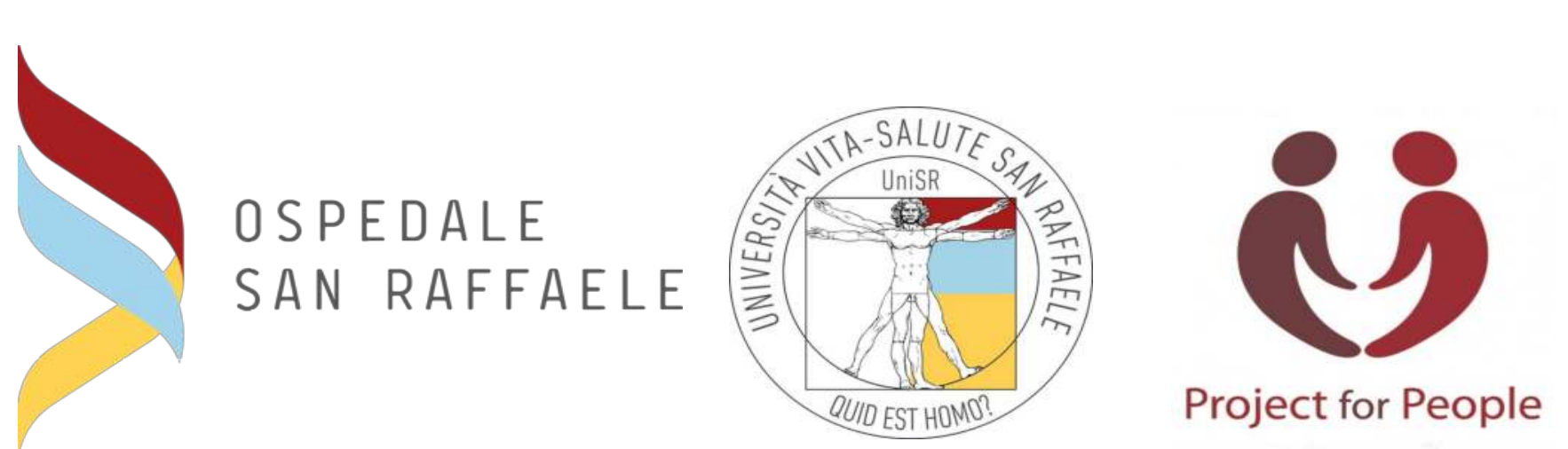

